\title{
Epigenetic regulation of hTERT promoter in hepatocellular carcinomas
}

\author{
DIMITRIOS ILIOPOULOS ${ }^{1}$, MARIA SATRA ${ }^{2}$, ALEXANDRA DRAKAKI ${ }^{3}$, \\ GEORGE A. POULTSIDES ${ }^{4}$ and ASPASIA TSEZOU ${ }^{1,2,5}$ \\ ${ }^{1}$ Institute of Biomedical Research and Technology, Larissa; ${ }^{2}$ University of Thessalia, \\ Medical School, Department of Biology, Larissa, Greece; ${ }^{3}$ St Elizabeth Medical \\ Center, Tufts University, Boston, MA; ${ }^{4}$ Department of Surgery, School of Medicine, \\ University of Connecticut, Farmington, CT, USA; ${ }^{5}$ University of Thessalia, Medical School, \\ Laboratory of Cytogenetics and Molecular Genetics, University Hospital of Larissa, Larissa, Greece
}

Received July 8, 2008; Accepted September 25, 2008

DOI: 10.3892/ijo_00000162

\begin{abstract}
Although hepatocellular carcinoma (HCC) is the fifth most common cancer worldwide, the molecular pathogenesis of the disease has not been elucidated. Several studies have shown that telomerase activity and hTERT expression are increased in HCCs. In the present study we tried to elucidate hTERT transcriptional and epigenetic regulatory mechanisms in HCC. hTERT expression was tested by real-time PCR and DNA methylation status was assessed by MethyLight and DNA bisulfite sequencing analyses in 106 tissues (64 with HCC and 42 without liver disorders) and also in 7 hepatocarcinoma cell lines (HepG2, HepG3B2, C3A, SNU-182, SNU-398, SBU-449 and SNU-475). hTERT expression levels were inversely correlated with DNA methylation levels in HCC and normal tissues ( $\mathrm{r}=-0.859)$. hTERT expression was found to be regulated by DNA methylation and histone H3-K9 modifications, affecting the ability of c-myc binding in E-box 1 site in hTERT promoter. Additionally, c-myc siRNA liposomal down-regulation inhibited significantly hTERT expression $(\mathrm{p}<0.05)$. Thus, we propose that hTERT is regulated by a combination of epigenetic mechanisms (DNA methylation and histone modifications) and by the transcription factor c-myc in HCC.
\end{abstract}

\section{Introduction}

Human hepatocellular carcinoma (HCC) is one of the most common solid tumors, rated fifth in incidence and third in mortality worldwide (1). Although much is known about the

Correspondence to: Dr Aspasia Tsezou, Department of Biology, School of Medicine, University of Thessalia, Mezourlo 41110, Larissa

E-mail: atsezou@med.uth.gr

Key words: telomerase, c-myc, DNA methylation, histone modifications etiological agents (i.e. $\mathrm{HBV}, \mathrm{HCV}$ infections) responsible for the majority of HCC, the molecular pathogenesis of HCC is not well understood (2). Activation of telomerase seems to be one of the molecular events that underlie the multigenetic process of hepatocarcinogenesis (3-5).

It has been recently described that telomerase consists of two molecules each of telomerase reverse transcriptase, telomerase RNA and dyskerin (6). hTERT is the catalytic component of telomerase and is considered to be the ratelimiting component of telomerase activity (7). The presence of telomerase activity as well as hTERT expression has been well documented in HCC (8-13).

It is known that hypermethylated promoters lack transcriptional activity, a fact which may lead to gene inactivation due to direct or indirect prevention to the access of transcription factors (14). CpG hypermethylation (also known as de novo methylation) acts as an alternative and/or complementary mechanism to gene mutations causing gene inactivation and it is now recognized as an important mechanism in carcinogenesis (15). Therefore, methylation profiles have been considered useful indicators of the transcriptional state of the genes in different types of cancer, including HCC $(16,17)$.

Regarding hTERT methylation status, previous studies have shown that it does not always correlate with hTERT expression levels in different types of cancer $(18,19)$, suggesting that other mechanisms such as histone modifications may regulate hTERT expression in these cells. Furthermore, several studies have shown that the oncogene c-myc acts as an important transcriptional regulator of hTERT since it binds to the DNA sequence 5'-CACGTG-3', termed E-box, which contains a CpG site (20-22).

In the present study, we attempted to elucidate the epigenetic and transcriptional regulation of hTERT in HCCs. Specifically, the aim was to determine whether hTERT methylation status is associated with hTERT expression and telomerase activity in normal and HCC liver tissues. In addition, we investigated how DNA methylation and histone modifications in hTERT promoter as well as c-myc, regulate hTERT expression in hepatocellular carcinomas. 


\section{Materials and methods}

Subjects. The study protocol conformed to the ethical guidelines of the University Hospital of Larissa. In this study the control group consisted of individuals without liver disease. Specifically, liver tissue specimens were obtained from 42 individuals (26 male, 16 female; mean age: 47.3 years, range $37-83$ years) during an operation that was performed for cholelithiasis (cholecystectomy). All individuals had apparently no evidence of chronic liver disease and normal ALT values $(24.5 \pm 5.1 \mathrm{U} / 1)$, tested negative for $\mathrm{HBsAg}$, anti-HCV and anti-HIV antibodies and denied ever having used hepatotoxic drugs, herbals, or having abused alcohol or injected drugs. The HCC tissue samples were purchased from US Biomax Inc cancer tissue bank collection (US Biomax Inc, MD, USA). From the 64 HCC samples, 43 were well differentiated (grade I-stage I; 11 due to HCV-related cirrhosis and 32 due to HBV-related cirrhosis) and 21 were moderately differentiated (grade II-stage II; all due to HCV-related cirrhosis). The diagnostic criteria for HCC were based on the conclusions of the Barcelona-2000 EASL conference (23) while the histological diagnosis was made according to the AJCC/UICC classification system (24).

Cell lines. HepG2, HepG3B2, C3A, SNU-182, SNU-398, SNU-449 and SNU-475 hepatocarcinoma cells were purchased from ATCC (USA). Cells were cultured in RPMI-1640 medium (Gibco, Paisley, Scotland) supplemented with $10 \%$ fetal bovine serum (Gibco), L-glutamin $2 \mathrm{mM}$ (Gibco), penicillin $100 \mathrm{IU} / \mathrm{ml}$ and streptomycin $100 \mu \mathrm{g} / \mathrm{ml}$ (Gibco) at $37^{\circ} \mathrm{C}$ in $5 \% \mathrm{CO}_{2}$.

TRAP assay. TRAP assay is considered a sensitive and specific PCR-based functional enzyme assay for determining telomerase activity. It was performed using TeloTAGGG telomerase PCR ELISA ${ }^{\text {PLUS }}$ kit (Roche, Indianapolis, USA), as described previously (8).

RNA extraction and real-time PCR analysis. RNA extraction and hTERT mRNA levels were assessed as described previously (8). For analysis of c-myc, the following probe and primers were used: Taq Man Probe, 6-FAM-5'-AGAAGCC GCTCCACATACAGTCCTGG-3'-TAMRA; forward primer, 5'-GGACGACGAGACCTTCATCAA-3'; and reverse primer, 5'-CCAGCTTCTCTGAGACGAGCTT-3'. To normalize hTERT and c-myc mRNA expression we amplified B-actin which served as loading control using the following primers and probes TGGTGATGGAGGAGGTTTAGTAAGT (forward), ACCAATAAAACCTACTCCTCCCTTAA (reverse), 6-FAM-ACCACCACCCAACACACAATAACA AACACA-TAMRA (probe).

Bisulfite treatment - MethyLight analysis. Bisulfite treatment was performed as described previously (25). DNA methylation was detected by a fluorescence-based, real-time PCR assay MethyLight. The following primers and probes for methylated DNA were used for the MethyLight reactions: a) hTERT: 5'-GCGTCGGAGGTTAAGGTTGTT-3', (forward primer), 5'-CTCTCCAAAATTACCGTACGCG-3' (reverse primer),
5'-6FAM-AACTCGCTCGCCCGCCGAA-BHQ-1-3' (probe). b) Actin: (primers and probe described above).

Bisulfite DNA sequencing. DNA was extracted from patient samples and bisulfite treated as described above. After bisulfite modification, PCR were performed with primers specifically designed to amplifiy bisulfite-modified DNA sequence of the promoter and first exon of hTERT 5'-CTAC CCCTTCACCTTCCAA-3' and 5'-GTTAGTTTTGGGGTT TTAGG-3'. Amplification was performed using the master mix (Promega), with the following PCR conditions: 40 cycles of $94^{\circ} \mathrm{C}$ for $30 \mathrm{sec}, 57^{\circ} \mathrm{C}$ for $45 \mathrm{sec}$ and $72^{\circ} \mathrm{C}$ for $50 \mathrm{sec}$. PCR products were cloned into the pGEM-T vector using the pGEM-T vector system II (Promega). DNA extracted from bacterial clones (QIAprep Spin Miniprep kit, Qiagen) was analyzed by sequencing with the M13 forward primer (5'-GTA AAACGACGGCCAG-3'), using a Big Dye Terminator Cycle Sequencing kit and an ABI Prism 3100 sequencer (Applied Biosystems, Foster City, CA).

Chromatin immunoprecipitation. The chromatin immunoprecipitation (ChIP) assay was done with a ChIP assay kit (Upstate Biotech) with anti-K9-dimethylated H3 and antiK9-acetylated H3 (Upstate Biotech) or c-myc (Ab56-AbCam Inc). Immunoprecipitated DNA was amplified by real-time PCR analysis. The primers used were as follows: hTERT promoter area sense 5'-GGCCGATTCGACCTCTCT-3' and hTERT promoter antisense 5'-AAGAAGCGGAACTGGA AGGT-3'. The primers used around E-box1 were: sense 5'-GGGCTCCCAGTGGATTC-3' and antisense 5'-CGGAG CTGGAAGGTGAAG-3'.

5-Aza-2-deoxycytidine and Trichostatin A treatment of hepatocellular carcinoma cells. SNU-449 cells treated with $5 \mu \mathrm{M}$ 5-Aza-2-deoxycytidine (DNA methylation inhibitor, Sigma) for 5 days and subsequently treated with $0.5 \mu \mathrm{M}$ of Trichostatin A (histone deacetylase inhibitor, Sigma) for 1 day.

Small interference RNA (siRNA) experiments. c-myc siRNA and negative control siRNA were purchased from Ambion, Inc (Austin, TX). HepG2 and SNU-449 $\left(2 \times 10^{5}\right)$ cells were grown in RPMI supplemented with $10 \%$ FBS and were transfected with hTERT or c-myc siRNA (50 nM) or siRNA scrambled as negative control using siPORT NeoFx lipid-based transfection agent (Ambion, Inc) in 6-well plates.

Cell growth analysis. Cells were counted in a Beckman Coulter Counter using vi-Cell viability program (analyzing 100 images). Cell growth was calculated as a percentage relative to untreated counterpart.

Luciferase assay. Transient transfections were done using Lipofectamine, according to the instructions of the manufacturer (Invitrogen, CA). DNA fragments (378, 31 and $3328 \mathrm{bp}$ ) upstream of the initiating ATG codon were PCR amplified and inserted into luciferase reporter vector pGL3Basic, a promoter- and enhancerless vector (Promega, CA) in sense orientation relative to the luciferase coding sequence at $M l u \mathrm{I}$ and $B g l \mathrm{II}$ sites. HepG2 cells were transfected with $0.5 \mu \mathrm{g}$ of the firefly luciferase reporter plasmid and $0.05 \mu \mathrm{g}$ of the 
A

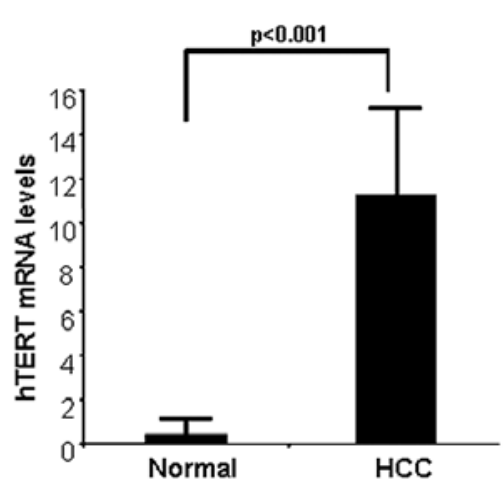

B

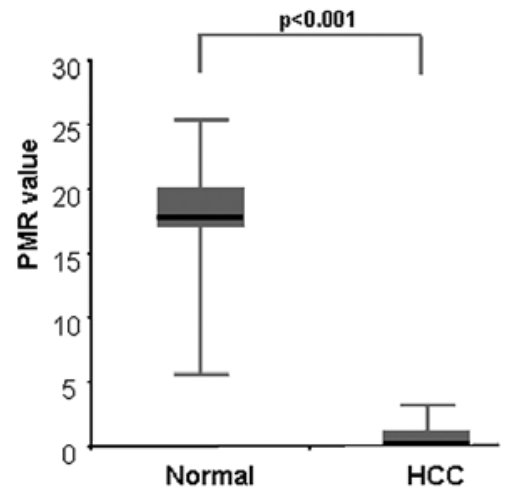

C

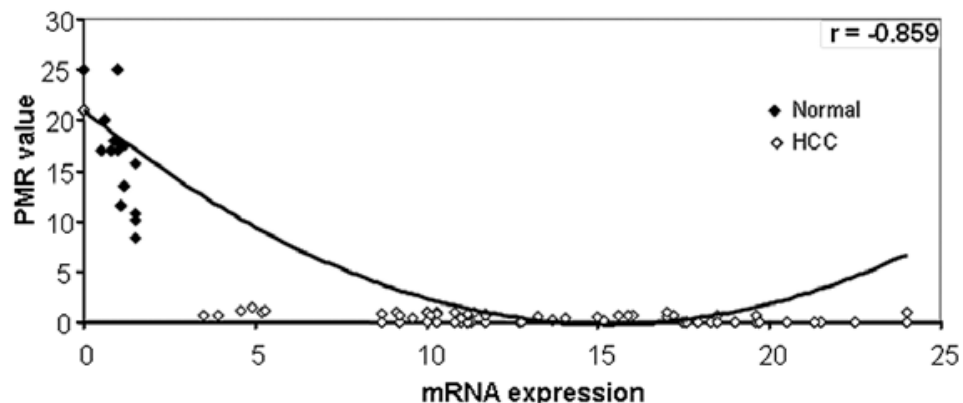

Figure 1. hTERT promoter methylation and expression levels are highly correlated in liver tissues. (A) Real-time PCR analysis revealed that hTERT expression was 10-fold higher in HCC compared to its expression in normal liver tissues $(\mathrm{p}<0.001)$. (B) MethyLight analysis showed that hTERT promoter was heavily methylated in normal liver tissue (mean PMR value: 17.6) and was hypomethylated in most of the hepatocellular carcinomas (mean PMR value: 0.8). (C) Correlation analysis showed strong correlation between hTERT promoter methylation and hTERT expression levels in HCC and normal liver tissues ( $\mathrm{r}=-0.8592$, $\mathrm{p}<0.001)$.

control plasmid containing Renilla luciferase, pRL-TK (Promega). Cells were lysed with passive lysis buffer and Firefly and Renilla luciferase activities were measured consecutively by using dual-luciferase assays (Promega) $24 \mathrm{~h}$ after transfection. Total DNA used in transfections was kept constant by adding parental vector. Transfections were performed in triplicate in 12-well plates.

Statistical analysis. Data were analysed by unpaired t-test, Mann-Whitney U (MWU), Kruskal-Wallis, as well as analysis of variance (ANOVA) and the Fisher PLSD as the post-hoc test corrected for multiple comparisons, where applicable. Correlation coefficients were calculated by simple regression analysis $(r)$ and non-parametric Spearman rank correlation $\left(r_{s}\right)$ when appropriate. Numerical data were expressed as mean \pm standard deviation $(\mathrm{SD})$. A two-sided p-value $<0.05$ was considered as statistically significant.

\section{Results}

hTERT is highly expressed in hepatocarcinomas in comparison to normal liver tissues. We assessed telomerase activity and hTERT mRNA expression in 64 HCCs and 42 normal liver samples. Telomerase activity (measured by TRAP) was not detected in control liver tissues, while it was strongly activated in HCCs specimens (mean levels 54.12 $\pm 8.73 \mathrm{U} / \mu \mathrm{g}$ protein). Quantification of hTERT mRNA revealed that the mean levels of hTERT mRNA expression were significantly $(\mathrm{p}<0.001)$ higher in HCC (11.21 \pm 4.57; range 2.12-24.67) compared to its expression in normal liver tissues $(\mathrm{p}<0.001$, Fig. 1A). Telomerase activity was correlated with hTERT mRNA expression levels $\left(\mathrm{r}^{2}=0.912, \mathrm{p}<0.001\right)$ in all liver tissues.

DNA methylation is a regulatory mechanism of hTERT expression in hepatocellular carcinomas. We were interested to investigate whether epigenetic mechanisms regulate hTERT expression in liver tissues. Using MethyLight analysis we found that there was a significant difference in hTERT promoter methylation levels between normal and cancer tissues (ANOVA, p<0.001). Specifically, we detected that hTERT promoter was heavily methylated in normal liver tissue (mean PMR value: 17.6) and was hypomethylated in most of the hepatocellular carcinomas (mean PMR value: 0.8 ) (Fig. 1B). These results suggest for the first time that DNA methylation is a major regulatory mechanism of hTERT expression in liver tissues. Furthermore, a strong inverse correlation was observed between hTERT promoter methylation and hTERT mRNA expression levels $(r=-0.859$, p<0.001) (Fig. 1C).

DNA bisulfite sequencing in HCC and normal tissues revealed a specific methylation pattern in hTERT promoter. We have shown that hTERT is regulated by DNA methylation in HCC and normal liver tissues by MethyLight analysis; however we were interested in studying in greater detail this regulatory mechanism in hTERT promoter by DNA bisulfite sequencing. 


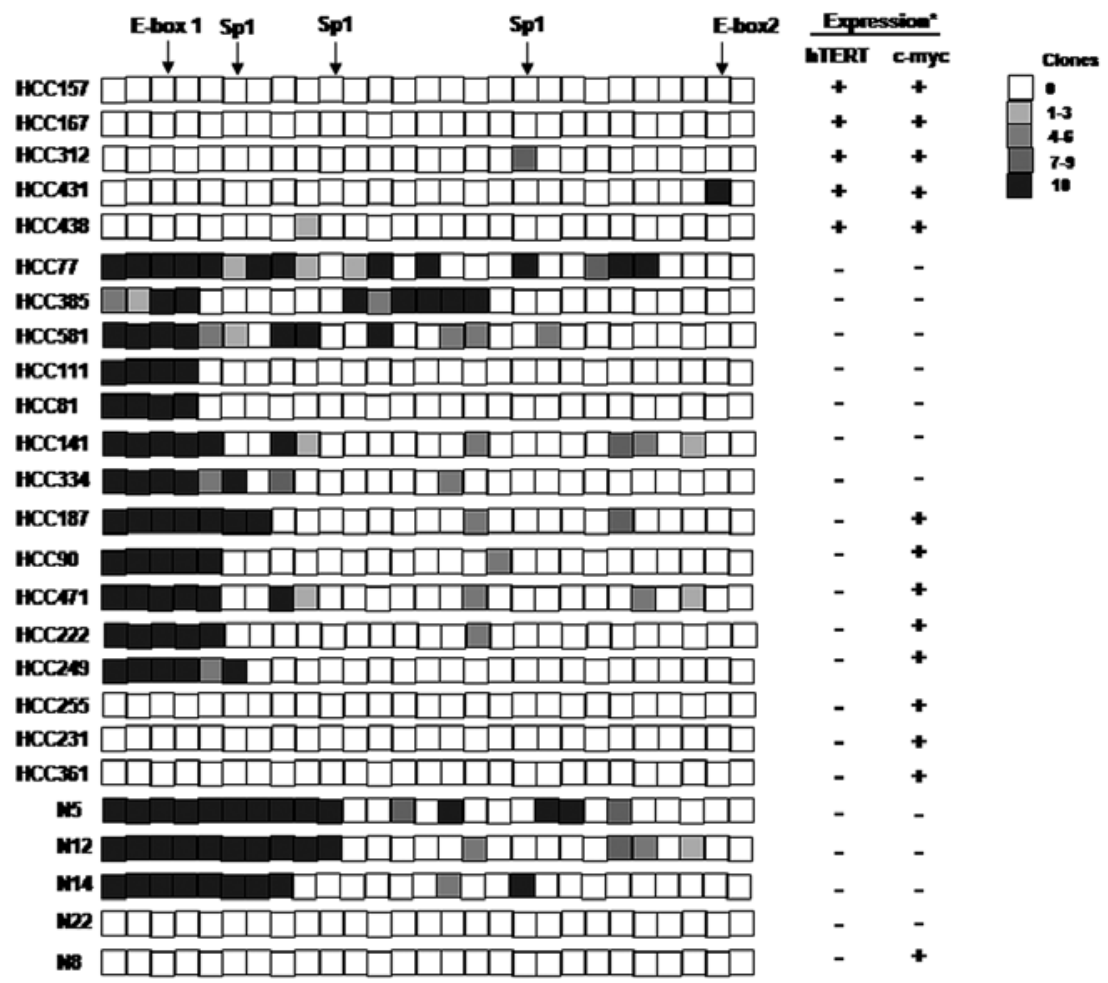

thERT and c-myc expression were evaluabd by real fime PCR

Figure 2. hTERT promoter methylation status assessed by DNA besulfite sequencing. Each square represents a CpG site (white means unmethylated and black means hypermathylated). hTERT promoter area consists of an E-box and $3 \mathrm{Sp1}$ sites included in CpG sites upstream of the transcription initiation site and a second E-box downstream the transcription initiation site $(+1)$. We performed DNA bisulfite sequencing in the whole region and for each sample we analyzed 10 clones. We found that in 52/64 (81\%) HCC tissues, hTERT was unmethylated and highly expressed, consistent with our results presented in Fig. 1B using MethyLight analysis. On the other hand, methylation in the first E-box CpG inhibited binding of c-myc (even when was highly expressed-for example HCC187) and hTERT was not expressed. In addition, we detected tissues were hTERT was not methylated and not expressed even in the presence of c-myc (e.g HCC255).

Thus, we analyzed by DNA bisulfite sequencing, the methylation status of $27 \mathrm{CpG}$ sites located in hTERT promoter area which structurally consists of two E-box areas, the first one in the position -165 to -160 bp relative to the site of the initiation of transcription and the second one in the position +44 to +49 . Between the 2 E-boxes are located 3 Sp1 binding sites (Fig. 2, white means unmethylated and black means hypermathylated). After sequencing 10 clones for each tissue sample we found that $81 \%(52 / 64)$ of HCC samples were completely unmethylated. We detected hypermethylation in 12 HCC samples (HCC77, HCC 385, HCC581, HCC111, HCC81, HCC141, HCC334, HCC187, HCC90, HCC471, HCC222, HCC249) where hTERT expression was downregulated (Fig. 2). In these samples a methylation pattern including E-box 1 binding site was clearly detected. On the other hand, most $(35 / 42)$ of the normal liver tissues presented hypermethylated hTERT promoter (representative results N5, N12, N14 shown in Fig. 2) and highly reduced hTERT expression. According to our data, we found that when hTERT is not methylated it is highly expressed (HCC157, HCC167, HCC312, HCC431, HCC438 are representative results for most of HCC samples). On the other hand, DNA hypermethylation in hTERT promoter (HCC77, HCC385, HCC581, HCC111, HCC81, HCC141, HCC334, N5, N12, N14) inhibited hTERT expression. In this group of samples, an interesting finding was that the presence of methylation pattern included always E-box1 CpG site, which is the binding site of c-myc.

Very interestingly, hTERT promoter methylation status did not always match with hTERT expression levels, suggesting the presence of another regulatory mechanism for hTERT expression in HCC. Previous studies have demonstrated that hTERT could be regulated epigenetically not only by DNA methylation but also by histone modifications. Consistent with these studies, we detected a group of tissues where hTERT promoter area was not methylated (HCC255, HCC231, HC361, N22, N8) and hTERT was not expressed.

DNA methylation and histone modifications regulate hTERT expression in hepatocarcinoma cell lines. In order to examine the possible involvement of chromatin modifications in the transcriptional regulation of hTERT, ChIP analysis in HepG2 (hTERT-positive), SNU-182 (hTERT-reduced) and SNU-449 (hTERT-negative) hepatocellular carcinoma cells was performed. More specifically, we examined the methylation and acetylation status of histone $\mathrm{H} 3$ lysine 9 (H3-K9) in hTERT promoter (same region as DNA methylation was tested). It is known that methylation of H3-K9 (H3-K9 Me) is a marker of condensed, inactive chromatin while $\mathrm{H} 3-\mathrm{K} 9$ acetylation (H3-K9 Ac) is associated with an open chromatin configuration as the one found in transcriptionally active promoters. We found that in HepG2 cells there is no DNA 
A

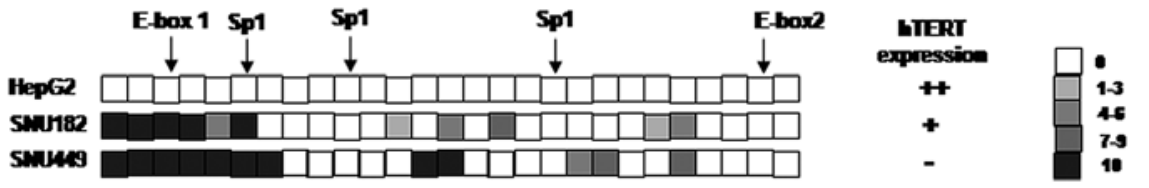

B

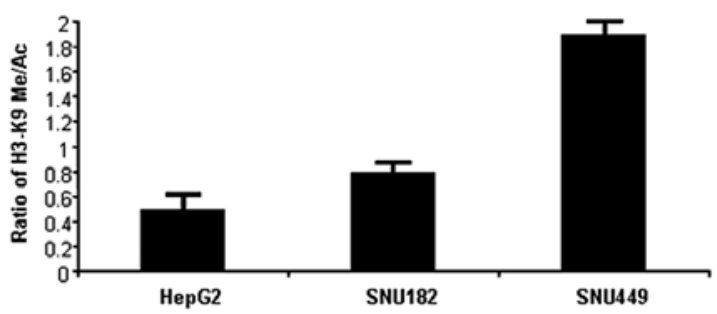

Figure 3. DNA methylation and histone modifications regulate hTERT expression in HCC cells. (A) hTERT is highly expressed (assessed by real-time PCR) and hypomethylated (detected by DNA bisulfite sequencing) in HepG2 cells, while in SNU182 and SNU449 is highly down-regulated or not expressed and hypermethylated. (B) Quantitation of ChIP PCR signals of methylation or acetylation of histone H3-K9. High ratio of H3-K9 Me/Ac is found in closed chromatin, while low ratio in open chromatin. In HepG2 and SNU182 cells we detected low ratio (0.5 and 0.8 respectively) of H3-K9 Me/Ac suggesting active chromatin around hTERT promoter while in SNU449 the ratio of H3-K9 Me/Ac was very high (1.9) suggesting a tight chromatin structure around hTERT promoter.

methylation in hTERT promoter and the ratio of $\mathrm{H} 3-\mathrm{K} 9$ $\mathrm{Me} / \mathrm{Ac}$ is very low in comparison with SNU-449 cells where hTERT promoter is DNA hypermethylated and the ratio of $\mathrm{H} 3-\mathrm{K} 9 \mathrm{Me} / \mathrm{Ac}$ is very high, suggesting a closed and dense chromatin state in hTERT promoter, which leads to its transcriptional inactivation (Fig. 3A and B). Furthermore, in SNU-182 cells, we detected DNA hypermethylation but the ratio of $\mathrm{H} 3-\mathrm{K} 9 \mathrm{Me} / \mathrm{Ac}$ was very low, suggesting an open chromatin state. These results suggest that hTERT can be regulated by DNA methylation and/or by histone modifications such as acetylation and methylation in hepatocellular carcinomas.

Reversal of epigenetic code re-activates hTERT in hepatocellular carcinoma cells. According to our results we found that hTERT is inactivated by promoter DNA methylation and histone modifications in SNU-449 cells. We treated these cells with the DNA methyltrasferase inhibitor, 5-Aza-2deoxycytidine (AZA) and with a histone deacetylase inhibitor, Trichostatin A (TSA), in order to reverse the epigenetic code in hTERT promoter and re-activate its expression. Specifically SNU-449 cells treated with $5 \mu \mathrm{M}$ AZA for 4 days and subsequently treated with $0.5 \mu \mathrm{M}$ of TSA for 1 day resulted in decreased DNA methylation levels (Fig. 4A) and H3-K9 $\mathrm{Me} / \mathrm{Ac}$ ratio (by increasing H3-K9 Ac levels) (Fig. 4B), reactivating hTERT expression.

c-myc is correlated with hTERT and regulates hepatocellular cancer cell growth. Above we have shown that hTERT is epigenetically regulated in hepatocellular carcinomas. c-myc is up-regulated in several cancers including hepatocellular carcinomas and binds in the E-box sites in hTERT promoter activating its expression (26). We were interested in studying how this transcription factor together with epigenetic mechanisms regulates hTERT expression in hepatocellular carcinomas. We tested if there is any correlation between c-myc and hTERT expression levels in hepatocarcinoma cell lines and specifically in HepG2, HepG3B2, C3A, SNU-182, SNU-398, SBU-449 and SNU-475 cell lines. We found that in cells with high c-myc expression, hTERT was also highly expressed (for example HepG2 cells), while in cells with low levels of c-myc, hTERT was also found down-regulated (for example SNU-182 cells) (Fig. 5A). The correlation rate between c-myc and hTERT expression levels was very high $\left(r^{2}=0.8126\right)$, suggesting a direct relationship between these two proteins in HCC cells. In order to understand better the relationship between c-myc and hTERT, we took advantage of RNAi technology and blocked c-myc expression in HepG2 cells using siRNA against c-myc transferred by liposomes. siRNA treatment against c-myc resulted in hTERT expression down-regulation (Fig. 5B). Specifically c-myc was $90 \%$ down-regulated $48 \mathrm{~h}$ post siRNA transfection, while hTERT was $60 \%$ down-regulated 72 post siRNA transfection. In addition c-myc inactivation decreased HepG2 cell growth rate 46.6 and $55.3 \%$ at days 3 and 4 respectively (Fig. 5C). All the experiments were controlled by transfecting scrambled siRNA as a control while no toxicity or effect on cancer cell growth (si mock) was detected.

Epigenetic mechanisms and c-myc orchestrate hTERT expression in hepatocellular cancer. c-myc should have access into E-box binding sites in hTERT promoter only when the chromatin is open (e.g when DNA is unmethylated and histones are acetylated), a hypothesis which is strengthened by our analysis in clinical samples (Fig. 2). Actually we found tissues (HCC187, HCC90, HCC471, HCC222, HCC249) positive for c-myc but negative for hTERT. In these tissues, c-myc probably can not bind to E-box 1 site due to its methylation status. 
A

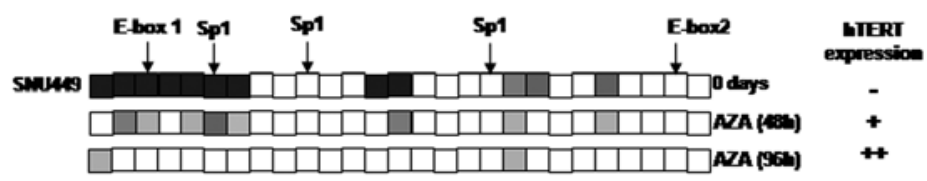

B

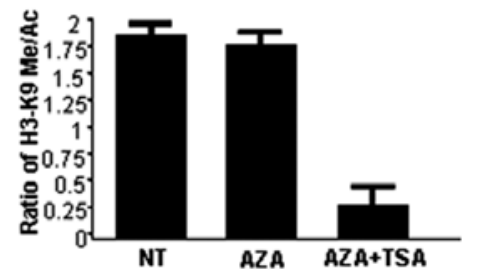

Figure 4. Reversal of hTERT epigenetic code activates its expression. 5-Aza-2-deoxycytidine and Trichostatin A re-activate hTERT expression in SNU449 cells. (A) Forty-eight hours of treatment with $5 \mu \mathrm{M}$ AZA decreased hTERT DNA methylation levels and actually $96 \mathrm{~h} 5 \mu \mathrm{M}$ AZA treatment demethylated completely hTERT promoter and activated hTERT expression (evaluated by real-time PCR analysis). (B) Consequent treatment with $0.5 \mu \mathrm{M}$ TSA reversed also the histone code by decreasing the ratio of $\mathrm{H} 3-\mathrm{K} 9 \mathrm{Me} / \mathrm{Ac}$.

A

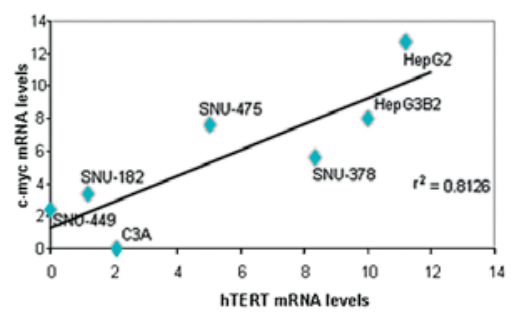

C

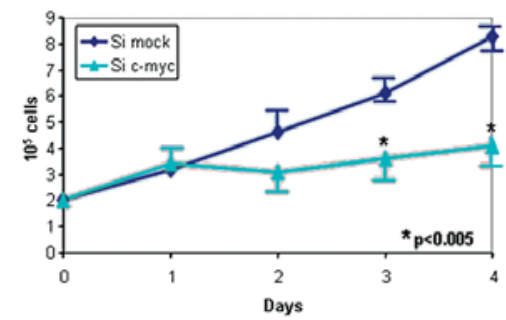

B

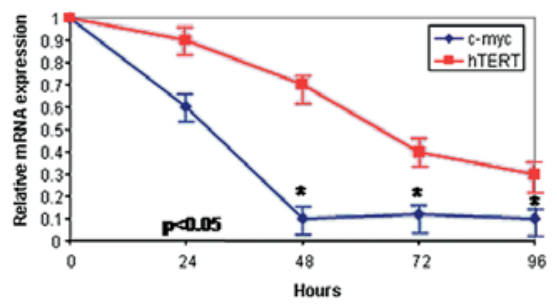

Figure 5. c-myc is a major regulator of hTERT expression and hepatocellular carcinoma cell growth. (A) High correlation rate $\left(\mathrm{r}^{2}=0.812\right)$ between $\mathrm{c}-\mathrm{myc}$ and hTERT expression levels in HepG2, HepG3B2, C3A, SNU-182, SNU-398, SBU-449 and SNU-475 hepatocellular carcinoma cells. (B) c-myc down-regulation by siRNA treatment $(50 \mathrm{nM})$ in HepG2 cells blocked hTERT expression and $(C)$ inhibited $\sim 50 \%$ cancer cell growth.

To verify this possibility experimentally, we overexpressed c-myc in HepG2 cells (E-box 1 site is unmethylated) and in SNU-449 cells (E-box 1 site is methylated). These two cell lines express in low levels c-myc and hTERT. Endogenous hTERT expression was up-regulated 8.1-fold in HepG2 cells while it was not significantly $(<1$-fold) up-regulated in SNU-449 cells (Fig. 6A), suggesting that E-box $1 \mathrm{CpG}$ site methylation inhibits c-myc from binding and activating hTERT.

In order to support these data we tested if c-myc is able to bind in an unmethylated or methylated E-box1. Chromatin immunoprecipitation revealed that endogenous c-myc was able to bind in umethylated E-box1 in HepG2 cells but not methylated E-box1 in SNU-449 cells (Fig. 6B). In Fig. 4A, we have shown that SNU-449 treatment with AZA $(5 \mu \mathrm{M})$ for 4 days is able to re-activate hTERT expression. Chromatin immunoprecipitation in SNU-449 cells revealed that c-myc was not able to bind in the methylated E-box 1 area of hTERT promoter (Fig. 6C, lane 1), while after 4 days of AZA treatment in the absence of DNA methylation (Fig. 6C, lane 2) c-myc was able to bind (9-fold enrichment over background).

Furthermore, there is a second E-box binding site for c-myc which was always found unmethylated in our tissue samples and its methylation status did not correlate with c-myc and hTERT expression status. In addition, c-myc was able to bind in the second E-box of both HepG2 and SNU449 cells (data not shown), suggesting that the first E-box is the c-myc primary site in hTERT promoter affecting its expression. 
A

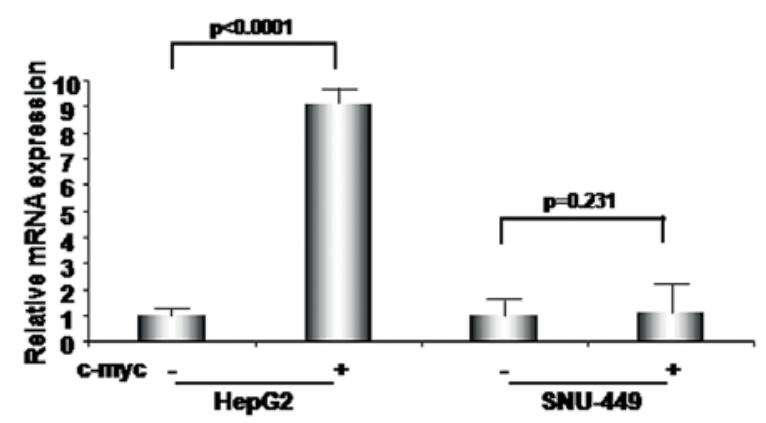

B

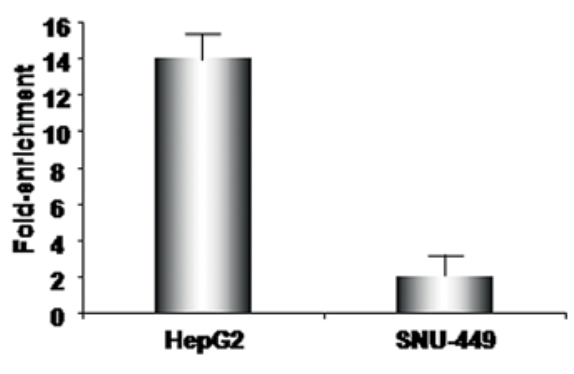

C

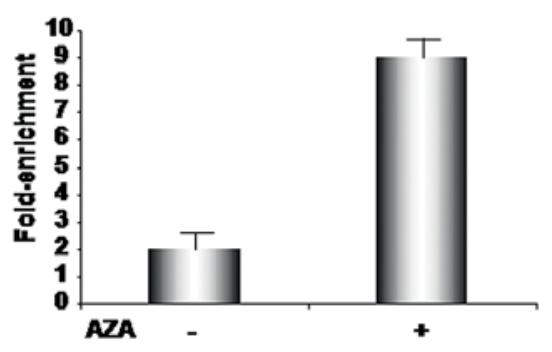

D

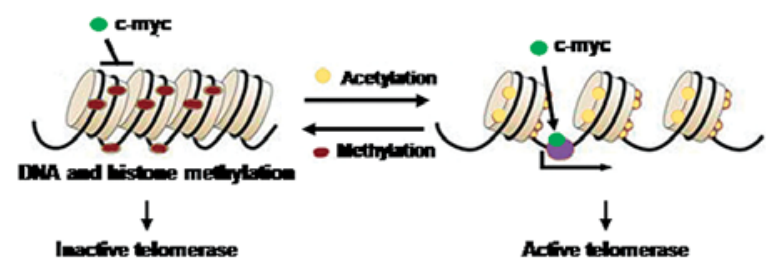

Figure 6. Chromatin modifications affect c-myc binding in hTERT promoter. (A) c-myc overexpression was able to up-regulate hTERT mRNA expression only in HepG2 cells (hypomethylated hTERT) and not in SNU449 cells (hypermethylated hTERT). (B) The ability of c-myc to bind to an unmethylated (HepG2) or methylated (SNU-449) E-box 1 in hTERT promoter was tested by chromatin immunoprecipitation. Immunoprecipitated DNA was quantified by real-time PCR analysis. (C) Treatment with AZA for 4 days in SNU-449 cells allowed c-myc binding in hTERT E-box 1 tested by chromatin immunoprecipitation. (D) Model of hTERT transcriptional regulation in hepatocellular carcinomas. hTERT is regulated by epigenetic mechanisms affecting c-myc binding ability to activate hTERT.

\section{Discussion}

A growing number of genes undergoing aberrant $\mathrm{CpG}$ island hypermethylation in HCC have been discovered, suggesting that de novo methylation is an important mechanism underlying malignant transformation in the liver (15,27-34). To date, there are no studies showing a correlation between hTERT DNA methylation and expression levels in HCCs and normal liver tissues.

In the present study, we observed a strong negative correlation between hTERT promoter methylation and hTERT expression in all liver tissues studied. Previous studies, which have tried to correlate hTERT methylation status and its expression levels in other human cancers, have revealed a discrepancy between hTERT methylation status and expression levels (19,35-37). Concretely, some studies have described a positive correlation between hTERT promoter regulation and methylation suggesting that hypermethylation correlates with high hTERT expression and telomerase activity, while other studies have shown no correlation between hTERT expression and the methylation status of its promoter (19,35-37). Detection of DNA methylation that correlates with expression levels of a specific gene, suggests the promoter area tested for DNA methylation is an important factor for gene transcription, probably due to transcription factor binding to this area. However, the discrepancies between DNA methylation and expression levels could be attributed to tumor heterogeneity (38).

C-myc is an important transcription factor that is frequently overexpressed in HCCs in comparison to normal tissues $(39,40)$. It has been reported that c-myc binds to two E-boxes located upstream and downstream the transcriptional initiation site in the hTERT promoter region (41), along with several other nuclear factors, such as Max and Mad $(26,42,43)$. We studied the correlation between c-myc and hTERT DNA methylation by assessing DNA methylation levels in a CG-rich area where c-myc potentially binds (E-boxes) as well as histone H3-K9 methylation and acetylation status. We found that methylation especially of the first E-box CpG site and/or histone H3-K9 methylation, inhibited binding of c-myc transcription factor and consequently hTERT expression in hepatocellular carcinomas.

It is known that most cancer cell lines and tumors achieve telomere maintenance by the reactivation of telomerase (44). Nevertheless, we detected several HCC tissue samples, where telomerase activity and hTERT mRNA levels were low or not expressed. In these tissues it can be suggested that telomere maintenance is achieved in the absence of telomerase by other mechanisms such as the alternative lengthening of telomere (ALT) mechanism (45). Of course other mechanisms may be regulating telomerase expression in hepatocellular carcinomas. Recently, it has been proposed that CTCF-binding regions in hTERT exon 1 regulate hTERT expression in carcinoma tissues and cells (46). In our study, we did not detect any difference in the methylation status of CTCF-binding region (data not shown), suggesting that probably CTCF is not essential for hTERT regulation in hepatocellular carcinomas.

We suggest, for the first time, that DNA methylation, histone modifications and c-myc could regulate hTERT expression in hepatocellular carcinomas (Fig. 6D). According to this model, when DNA is hypermethylated and histone H3-K9 is methylated, c-myc binding in E-box 1 is inhibited, 
a fact which inactivates hTERT expression. On the other hand, when histone H3-K9 are acetylated and hTERT promoter is not methylated, c-myc is able to bind and activate hTERT expression. All these results propose the complexity of transcriptional regulation of hTERT. It seems that different combinations of transcription factors and epigenetic mechanisms regulate hTERT gene regulation, suggesting that hTERT transcriptional regulation is cancer cell type specific.

\section{References}

1. Parkin DM, Bray F, Ferlay J and Pisani P: Global cancer statistics, 2002. CA Cancer J Clin 55: 74-108, 2005

2. Nowak AK, Chow PK and Findlay M: Systemic therapy for advanced hepatocellular carcinoma: A review. Eur J Cancer 40: 1474-1484, 2004.

3. Okuda K: Hepatocellular carcinoma. J Hepatol 32: 225-237, 2000.

4. Moradpour D and Blum HE: Pathogenesis of hepatocellular carcinoma. Eur J Gastroenterol Hepatol 17: 477-483, 2005.

5. Lechel A, Manns MP and Rudolph KL: Telomeres and telomerase: new targets for the treatment of liver cirrhosis and hepatocellular carcinoma. J Hepatol 41: 491-497, 2004.

6. Cohen SB, Graham ME, Lovrecz GO, Bache N, Robinson PJ and Reddel RR: Protein composition of catalytically active human telomerase from immoral cells. Science 315: 1850-1855, 2007.

7. Theise ND, Park YN and Kojiro M: Dysplastic nodules and hepatocarcinogenesis. In: Clinics in Liver Disease. Liver histopathology. 1st edition. W.B. Saunders, Philadelphia, 2002.

8. Satra M, Gatselis N, Iliopoulos D, Zacharoulis D, Dalekos GN and Tsezou A: Real time quantification of human telomerase reverse transcriptase mRNA in liver tissues from patients with hepatocellular cancer and chronic viral hepatitis. J Viral Hepat 14: 41-47, 2007.

9. Oh BK, Kim YJ, Park YN, Choi J, Kim KS and Park C: Quantitative assessment of hTERT mRNA expression in dysplastic nodules of HBV-related hepatocarcinogenesis. Am J Gastroenterol 101: 831-838, 2006.

10. Zhang X, Dong N, Zhang H, You J, Wang H and Ye L: Effects of hepatitis $\mathrm{B}$ virus $\mathrm{X}$ protein on human telomerase reverse transcriptase expression and activity in hepatoma cells. J Lab Clin Med 145: 98-104, 2005.

11. Miura N, Maruyama S, Oyama K, Horie Y, Kohno M, Noma E, Sakaguchi S, Nagashima M, Kudo M, Kishimoto Y, Kawasaki H, Hasegawa J and Shiota G: Development of a novel assay to quantify serum human telomerase reverse transcriptase messenge RNA and ist significance asa tumor marker for hepatocellular carcinoma. Oncology 72: 45-51, 2007.

12. Shimojima M, Komine F, Hisatomi H, Shimizu T, Moriyama M and Arakawa Y: Detection of telomerase activity, telomerase RNA component, and telomerase reverse transcriptase in human hepatocellular carcinoma. Hepatol Res 29: 31-38, 2004.

13. Harada K, Yasoshima M, Ozaki S, Sanzen T and Nakanuma Y: PCR and in situ hybridization studies of telomerase subunits in human non-neoplastic livers. J Pathol 193: 210-221, 2001.

14. Adrien LR, Schlecht NF, Kawachi N, Smith RV, Brandwein-Gensler M, Massimi A, Chen S, Prystowsky MB, Childs G and Belbin TJ: Classification of DNA methylation patterns in tumor cell genomes using a $\mathrm{CpG}$ island microarray. Cytogenet Genome Res 114: 16-23, 2006.

15. Calvisi DF, Ladu S, Gorden A, Farina M, Lee JS, Conner EA Schroeder I, Factor VM and Thorgeirsson SS: Mechanistic and prognostic significance of aberrant methylation in the molecular pathogenesis of human hepatocellular carcinoma. J Clin Invest 117: 2713-2722, 2007

16. Herath NI, Leggett BA and MacDonald GA: Review of genetic and epigenetic alterations in hepatocarcinogenesis. J Gastroenterol Hepatol 21: 15-21, 2006.

17. Lee S, Lee HJ, Kim JH, Lee HS, Jang JJ and Kang GH: Aberrant $\mathrm{CpG}$ island hypermethylation along multistep hepatocarcinogenesis. Am J Pathol 163: 1371-1378, 2003.

18. Widschwendter A, Müller HM, Fiegl H, Ivarsson L, Wiedemair A, Müller-Holzner E, Goebel G, Marth C and Widschwendter M: DNA methylation in serum and tumors of cervical cancer patients. Clin Cancer Res 10: 565-571, 2004.
19. Guilleret I and Benhattar J: Demethylation of the human telomerase catalytic subunit (hTERT) gene promoter reduced hTERT expression and telomerase activity and shortened telomeres. Exp Cell Res 289: 326-334, 2003.

20. Cerni C: Telomeres, telomerase and myc. An update. Mutat Res 462: 31-47, 2000.

21. Hung L and Kumar V: Antisense regulation of expression and transactivation functions of the tumorigenic $\mathrm{HBx}$ and c-myc genes. Biochem Biophys Res Commun 344: 293-299, 2006.

22. Cheng J, Luo J, Zhang X, Hu J, Hui H, Wang C and Stern A: Inhibition of cell proliferation in HCC-9204 hepatoma cells by a c-myc specific ribozyme. Cancer Gene Ther 7: 407-412, 2000.

23. Bruix J, Sherman M, Llovet JM, Beaugrand M, Lencioni R, Burroughs AK, Christensen E, Pagliaro L, Colombo M and Rodés J: Clinical management of hepatocellular carcinoma. Conclusions of the Barcelona-2000 EASL conference. J Hepatol 35: 421-430, 2001

24. Green FL, Page DL and Fleming ID: American Joint Committee on Cancer. In: Liver (including intrahepatic bile ducts). AJCC Cancer Staging Handbook. 6th edition. Springer, New York, pp121-144, 2002.

25. Iliopoulos D, Guler G, Han SY, Johnston D, Druck T, McCorkell KA, Palazzo J, McCue PA, Baffa R and Huebner K: Fragile genes as biomarkers: epigenetic control of WWOX and FHIT in lung, breast and bladder cancer. Oncogene 24: 1625-1633, 2005.

26. Wu KJ, Grandori C, Amacker M, Simon-Vermot N, Polack A, Lingner $J$ and Dalla-Favera R: Direct activation of TERT transcription c-myc. Nat Genet 21: 220-224, 1999.

27. Calvisi DF, Ladu S, Gorden A, Farina M, Conner EA, Lee JS, Factor VM and Thorgeirsson SS: Ubiquitous activation of Ras and Jak/Stat pathways in human HCC. Gastroenterology 130: 1117-1128, 2006

28. Jicai Z, Zongtao Y, Jun L, Haiping L, Jianmin W and Lihua H: Persistent infection of hepatitis B virus is involved in high rate of p16 methylation in hepatocellular carcinoma. Mol Carcinog 45: 530-536, 2006.

29. Yang B, Guo M, Herman JG and Clark DP: Aberrant promoter methylation profiles of tumor suppressor genes in hepatocellular carcinoma. Am J Pathol 163: 1101-1107, 2003.

30. Yu J, Ni M, Xu J, Zhang H, Gao B, Gu J, Chen J, Zhang L, Wu M, Zhen S and Zhu J: Methylation profiles of twenty promoter-CpG islands of genes which may contribute to hepatocellular carcinogenesis. BMC Cancer 2: 29, 2002.

31. Yu J, Zhang HY, Ma ZZ, Lu W, Wang YF and Zhu JD: Methylation profiling of twenty four genes and the concordant methylation behaviors of nineteen genes that may contribute to hepatocellular carcinogenesis. Cell Res 13: 319-333, 2003.

32. Song J, Kim CJ, Cho YG, Kim SY, Nam SW, Lee SH, Yoo NJ, Lee JY and Park WS: Genetic and epigenetic alterations of the KLF6 gene in hepatocellular carcinoma. J Gastroenterol Hepatol 21: 1286-1289, 2006

33. Nishida N, Nagasaka T, Nishimura T, Ikai I, Boland CR and Goel A: Aberrant methylation of multiple tumor suppressor genes in aging liver, chronic hepatitis, and hepatocellular carcinoma. Hepatology 47: 908-918, 2008.

34. Nomoto S, Kinoshita T, Kato K, Otani S, Kasuya H, Takeda S, Kanazumi N, Sugimoto H and Nakao A: Hypermethylation of multiple genes as clonal markers in multicentric hepatocellular carcinoma. Br J Cancer 97: 1260-1265, 2007.

35. Dessain SK, Yu H, Reddel RR, Beijersbergen RL and Weinberg RA: Methylation of the human telomerase gene $\mathrm{CpG}$ island. Cancer Res 60: 537-541, 2000.

36. Kumakura S, Tsutsui TW, Yagisawa J, Barrett JC and Tsutsui T: Reversible conversion of immortal human cells from telomerase-positive to telomerase-negative cells. Cancer Res 65: 2778-2786, 2005

37. Widschwendter A, Müller HM, Hubalek MM, Wiedemair A, Fiegl H, Goebel G, Mueller-Holzner E, Marth C and Widschwendter M: Methylation status and expression of human telomerase reverse transcriptase in ovarian and cervical cancer. Gynecol Oncol 93: 407-416, 2004.

38. Feitelson MA: c-myc overexpression in hepatocarcinogenesis. Hum Pathol 35: 1299-1302, 2004.

39. Liu YC, Chen CJ, Wu HS, Chan DC, Yu JC, Yang AH, Cheng YL, Lee SC and Harn HJ: Telomerase and c-myc expression in hepatocellular carcinomas. EJSO 30: 384-390, 2004. 
40. Romano PR, McCallus DE and Pachuk CJ: RNA interferencemediated prevention and therapy for hepatocellular carcinoma. Oncogene 25: 3857-3865, 2006.

41. Takakura M, Kyo S and Kanaya T: Cloning of human telomerase catalytic subunit (hTERT) gene promoter and identification of proximal core promoter sequences essential for transcriptional activation in immortalized and cancer cells. Cancer Res 59: 551-557, 1999.

42. Wu D, Popov N, Hou M, Wang Q, Björkholm M, Gruber A, Menkel AR and Henriksson M: Switch from Myc/Max to Mad1/Max binding and decrease in histone acetylation at the telomerase reverse transcriptase promoter during differentiation of HL60 cells. Proc Natl Acad Sci USA 98: 3826-3831, 2001.

43. Lebel R, McDuff FO, Lavigne P and Grandbois M: Direct visualization of the binding of $\mathrm{c}-\mathrm{Myc} / \mathrm{Max}$ heterodimeric $\mathrm{b}-\mathrm{HLH}$ $\mathrm{LZ}$ to E-box sequences on the hTERT promoter. Biochemistry 46: 10279-10286, 2007
44. Holt SE and Shay JW: Role of telomerase in cellular proliferation and cancer. J Cell Physiol 180: 10-18, 1999.

45. Bryan TM, Englezou A, Gupta J, Bacchetti S and Reddel RR Telomere elongation in immortal human cells without detectable telomerase activity. EMBO J 14: 4240-4248, 1995.

46. Renaud S, Loukinov D, Abdullaev Z, Guilleret I, Bosman FT, Lobanenkov V and Benhattar J: Dual role of DNA methylation inside and outside of CTCF-binding regions in the transcriptional regulation of the telomerase hTERT gene. Nucleic Acids Res 35: 1245-1256, 2007 